\title{
Second-Order Cone Programming Approach to Design of Linkage Mechanisms With Arbitrarily Inclined Hinges
}

\author{
$\operatorname{AUTHOR}(S)$ :
}

Ohsaki, Makoto; Kanno, Yoshihiro; Yamaoka, Yuki

\section{CITATION:}

Ohsaki, Makoto ... [et al]. Second-Order Cone Programming Approach to Design of Linkage Mechanisms With Arbitrarily Inclined Hinges. Journal of Mechanical Design 2018, 140(10): 102301.

\section{ISSUE DATE:}

2018-07-30

URL:

http://hdl.handle.net/2433/236062

\section{RIGHT:}

Copyright $\odot 2018$ by ASME. This manuscript version is made available under the CC-BY-NC-ND 4.0 license

http://creativecommons.org/licenses/by-nc-nd/4.0/.; The full-text file will be made open to the public on 30 July 2019 in accordance with publisher's 'Terms and Conditions for Self-Archiving'., この論文は出版社版でありません。引用の際 には出版社版をご確認ご利用ください。; This is not the published version. Please cite only the published version. 


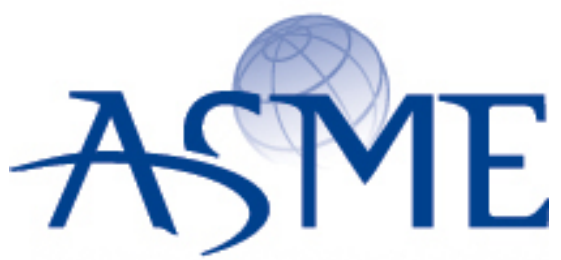

\section{American Society of Mechanical Engineers}

SETTING THE STANDARD

\section{ASME Accepted Manuscript Repository}

\section{Institutional Repository Cover Sheet}

First

Last

ASME Paper Title:

Second-Order Cone Programming Approach to Design of

Linkage Mechanisms With Arbitrarily Inclined Hinges

Authors:

Ohsaki Makoto, Kanno Yoshihiro, Yamaoka Yuki

ASME Journal Title: Journal of Mechanical Design

Volume/Issue

Vol. 140, Issue 10

Date of Publication (VOR* Online)

July 302018

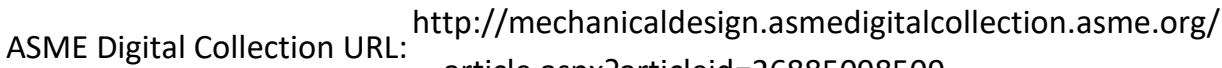

article. aspx ?articleid $=26885098509$

DOI:

*VOR (version of record) 


\title{
Second-Order Cone Programming Approach to Design of Linkage Mechanisms with Arbitrarily Inclined Hinges
}

\author{
Makoto Ohsaki ${ }^{1}$ \\ Department of Architecture and Architectural Engineering, Kyoto University \\ Kyoto-Daigaku Katsura, Nishikyo, Kyoto 615-8540, Japan \\ ohsaki@archi.kyoto-u.ac.jp \\ Yoshihiro Kanno \\ Mathematics and Informatics Center, The University of Tokyo \\ Hongo 7-3-1, Bunkyo, Tokyo 113-8656, Japan \\ kanno@mist.i.u-tokyo.ac.jp

\section{Yuki Yamaoka} \\ Department of Architecture and Architectural Engineering, Kyoto University \\ Kyoto-Daigaku Katsura, Nishikyo, Kyoto 615-8540, Japan \\ y.yamaoka28@gmail.com
}

\begin{abstract}
An optimization approach is presented for generating linkage mechanisms consisting of frame members with arbitrarily inclined hinges. A second-order cone programming (SOCP) problem is solved to obtain the locations and directions of hinges of an infinitesimal mechanism. It is shown that the primal and dual SOCP problems correspond to the plastic limit analysis problems based on the lower-bound and upperbound theorems, respectively, with quadratic yield functions. Constraints on displacement components are added to the dual problem, if a desirable deformation is not obtained. A finite mechanism is generated by carrying out geometrically nonlinear analysis and, if necessary, adding hinges and removing members. Effectiveness of the proposed method is demonstrated through examples of 2- and 3-dimensional mechanisms.
\end{abstract}

\footnotetext{
${ }^{1}$ Corresponding author
} 
Journal of Mechanical Design

\section{INTRODUCTION}

Design of linkage mechanisms is extensively studied for application to structures and machines in various fields of engineering [1]. Deployable structures and transformable structures are used for temporary shelters and roofs [2, 3]. Scissor structures are often used as basic one-dimensional mechanisms for deployable structures [4], which can be extended to two-dimensional mechanisms [5]. Synthesis problem of mechanism can often be formulated as a numerical optimization problem [6-9]. The design problem of types and numbers of linkages can be formulated as topology optimization problems [10-13]. However, most of the existing numerical approaches can be applied only to planar mechanisms. Kim et al. [14] proposed an optimization approach to generating 3dimensional mechanisms using a truss ground structure approach with springs at joints. Global mechanism is assured based on work transmittance efficiency [15].

In order to extend the methods for planar mechanisms to 3-dimensional mechanisms, it is important to consider a mechanism with partially rigid joints such as revolute and screw joints. Ohsaki et al. [16] and Tsuda et al. [17] presented a design method of 3-dimensional mechanisms of partially rigid frames based on plastic limit analysis. However, in their method, the hinges are restricted to rotate around specified axes in the orthogonal directions of local coordinates. Ohsaki et al. [18] demonstrated that a mechanism with arbitrary inclined hinges can be obtained by solving a quadratic programming (QP) problem based on the lower-bound theorem of limit analysis. However, in our previous research, a mechanism with local deformation is often found, and it is difficult to prevent the existence of local modes of deformation using the 
Journal of Mechanical Design

formulation based on the lower-bound theorem. Therefore, it is desirable to use a dual formulation assigning displacement constraints. However, if a QP approach is used, the variables of the primal problem based on the lower bound theorem and the dual problem based on the upper bound theorem cannot be separated clearly, when displacement constraints are assigned.

In this paper, we present a numerical method based on the second-order cone programming (SOCP), which is a class of convex optimization problems, and includes the linear programming, $Q P$, etc. It is known that SOCP can be solved efficiently with a primal-dual interior-point method; see $[19,20]$ for fundamentals of SOCP. Recently, application of SOCP to diverse problems in engineering mechanics has become popular. Particularly, it has been recognized that approaches based on SOCP are efficient for problems in computational plasticity, including the limit analysis, shakedown analysis, and incremental analysis; see, e.g., Refs. [21-26]. As for applications to geometrically nonlinear problems, the reader may refer to Refs. [27, 28].

In this paper, we first formulate an SOCP problem that is equivalent to a limit analysis problem, based on the lower-bound theorem, with quadratic yield functions with respect to member-end moments. The dual problem is then derived through the formulation of SOCP. This dual problem is regarded as a limit analysis problem based on the upper-bound theorem, and at the same time, it is regarded as a problem of solving an under-determined linear equations with the sum of some $L_{2}$-norm penalty terms. Constraints on displacement components are added if a desirable deformation is not obtained. A finite mechanism is generated by carrying out geometrically nonlinear 
Journal of Mechanical Design

analysis and adding hinges, if necessary. Effectiveness of the proposed method is demonstrated through numerical examples of 2- and 3-dimensional mechanisms.

\section{DEFINITION OF VARIABLES}

A limit analysis problem is solved to find the locations and directions of plastic hinges of a rigidly-jointed frame that can have perfectly elastoplastic hinges at member-ends. Plastic hinges are replaced with revolute joints that can rotate without external load to generate an infinitesimal mechanism of a linkage [16-18].

Figure 1(a) describes the local member coordinates of member $k$ with two nodes (member-ends) $a$ and $b$. The global coordinates are denoted by ( $x, y, z)$. The axial force in the direction of axis 1 and the torsional moment around axis 1 are denoted by $N^{(k)}$ and $T^{(k)}$, respectively. The bending moments are denoted by $M_{j 2}^{(k)}$ and $M_{j 3}^{(k)}$, as shown in Fig. 1(b), at node $j \in\{a, b\}$. The member-end shear forces are eliminated as functions of bending moments using the equilibrium equations to reduce the number of components of member-end forces to six. Formulations of equilibrium equations and compatibility conditions are briefly described below. See Ref. [14] for details.

Let $\mathbf{f}=\left(f_{1}, \ldots, f_{6 m}\right)^{\mathrm{T}}$ denote the vector of member forces consisting of $N^{(k)}, M_{a 2}^{(k)}$, $M_{a 3}^{(k)}, M_{b 2}^{(k)}, M_{b 3}^{(k)}$, and $T^{(k)}(k=1,2, \ldots, m)$. The nodal load vector is denoted by $\mathbf{p}=\left(p_{1}, \ldots, p_{n}\right)^{\mathrm{T}}$, where $n$ is the number of degrees of freedom (DOFs) after removing the fixed DOFs. The equilibrium equation is written as follows using the equilibrium matrix $\mathbf{H}=\left(\mathbf{h}_{1}, \ldots, \mathbf{h}_{6 m}\right) \in \mathbb{R}^{n \times 6 m}$ consisting of the direction cosines of the local axes: 
Journal of Mechanical Design

$$
\mathbf{H f}=\mathbf{p}
$$

The generalized member strain vector is denoted by $\mathbf{c}=\left(c_{1}, \ldots, c_{6 m}\right)^{\mathrm{T}}$, where $c_{i}$ corresponds to member extension and member-end rotations around axes $1,2,3$, respectively, if $f_{i}$ is axial force, torsional moment, and bending moments around axes 2 and 3. Relation between $\mathbf{c}$ and the nodal displacement vector $\mathbf{u}=\left(u_{1}, \ldots, u_{n}\right)^{\mathrm{T}}$ is defined using the compatibility matrix $\mathbf{H}^{\mathrm{T}}$ as

$$
\mathbf{c}=\mathbf{H}^{\mathrm{T}} \mathbf{u}
$$

\section{SOCP PROBLEMS FOR GENERATION OF LINKAGE MECHANISMS}

We find an infinitesimal mechanism such that the output node moves in the specified direction as the result of assigning the specified displacement at the input node in the specified direction. For this purpose, a limit analysis problem with a quadratic yield function is formulated.

Vectors of member-end moments $\mathbf{m}_{a}^{(k)}$ and $\mathbf{m}_{b}^{(k)}$ are defined as

$$
\mathbf{m}_{a}^{(k)}=\left(-T^{(k)}, M_{a 2}^{(k)}, M_{a 3}^{(k)}\right)^{\mathrm{T}}, \quad \mathbf{m}_{b}^{(k)}=\left(T^{(k)}, M_{b 2}^{(k)}, M_{b 3}^{(k)}\right)^{\mathrm{T}}
$$

The variables $N^{(k)}, \mathbf{m}_{a}^{(k)}$, and $\mathbf{m}_{b}^{(k)}$ are expressed in terms of $\mathbf{f}, \mathbf{C}_{0}^{(k)} \in \mathbb{R}^{1 \times 6 m}$, $\mathbf{C}_{a}^{(k)} \in \mathbb{R}^{3 \times 6 m}$, and $\mathbf{C}_{b}^{(k)} \in \mathbb{R}^{3 \times 6 m}$ as

$$
N^{(k)}=\mathbf{C}_{0}^{(k)} \mathbf{f}, \quad \mathbf{m}_{a}^{(k)}=\mathbf{C}_{a}^{(k)} \mathbf{f}, \quad \mathbf{m}_{b}^{(k)}=\mathbf{C}_{b}^{(k)} \mathbf{f}
$$

Each row of $\mathbf{C}_{0}^{(k)}, \mathbf{C}_{a}^{(k)}$, and $\mathbf{C}_{b}^{(k)}$ has one non-zero component 1 or -1 . The details are explained below using their condensed forms. 
Journal of Mechanical Design

The load vectors that have nonzero values in the components corresponding to the input and output DOFs, respectively, and 0 in other components are denoted by $\mathbf{p}_{\text {in }}$ and $\mathbf{p}_{\text {out }}$. The SOCP problem for maximizing the load coefficient $\lambda_{\text {in }}$ corresponding to the input load in the presence of output load is formulated as follows:

$$
\begin{array}{ll}
\underset{\lambda_{\text {in }}, \mathbf{f}}{\operatorname{maximize}} & \lambda_{\text {in }} \\
\text { subject to } & \mathbf{H f}=\mathbf{p}_{\text {out }}+\lambda_{\text {in }} \mathbf{p}_{\text {in }} \\
& \left\|\mathbf{C}_{j}^{(k)} \mathbf{f}\right\| \leq \alpha w_{\mathrm{m}}, \quad(k=1, \ldots, m ; j \in\{a, b\}) \\
& \left|\mathbf{C}_{0}^{(k)} \mathbf{f}\right| \leq \alpha w_{\mathrm{f}}, \quad(k=1, \ldots, m)
\end{array}
$$

where $w_{\mathrm{m}}$ and $w_{\mathrm{f}}$ are the weight coefficients for the fully plastic moment and the yield axial force, respectively, and $\alpha$ is a scaling parameter. The load factor $\lambda_{\text {in }}$ and the member-end force vector $\mathbf{f}$ are to be determined by solving problem (5); therefore, the problem has a linear objective function, linear equality and inequality constraints, and second-order cone constraints.

The dual problem of problem (5) is derived based on the standard approach of SOCP. The Lagrange multipliers of the equality constraints, which are the equilibrium equations, are the displacements $\mathbf{u}$. Let $\kappa_{0}^{(k)}, \quad \boldsymbol{\kappa}_{a}^{(k)}=\left(\kappa_{a 1}^{(k)}, \kappa_{a 2}^{(k)}, \kappa_{a 3}^{(k)}\right)^{\mathrm{T}}$, and $\boldsymbol{\kappa}_{b}^{(k)}=\left(\boldsymbol{\kappa}_{b 1}^{(k)}, \boldsymbol{\kappa}_{b 2}^{(k)}, \boldsymbol{\kappa}_{b 3}^{(k)}\right)^{\mathrm{T}}$ denote the Lagrange multipliers for the inequality constraints in problem (5). Then, the dual problem of problem (5), which is also an SOCP problem, is obtained as follows: 
Journal of Mechanical Design

$$
\begin{aligned}
\underset{\substack{\mathbf{u}, \kappa_{0}^{(1)}, \ldots, \kappa_{0}^{(m)}, \mathbf{\kappa}_{a}^{(1)}, \ldots, \mathbf{\kappa}_{a}^{(m)}, \mathbf{\kappa}_{b}^{(1)}, \ldots, \mathbf{\kappa}_{b}^{(m)}}}{\operatorname{minimize}}-\mathbf{p}_{\text {out }}^{\mathrm{T}} \mathbf{u}+\sum_{k=1}^{m} \alpha w_{\mathrm{f}}\left|\boldsymbol{\kappa}_{0}^{(k)}\right|+\sum_{k=1}^{m} \alpha w_{\mathrm{m}}\left\|\boldsymbol{\kappa}_{a}^{(k)}\right\|+\sum_{k=1}^{m} \alpha w_{\mathrm{m}}\left\|\mathbf{\kappa}_{b}^{(k)}\right\| \\
\text { subject to } \mathbf{p}_{\text {in }}^{\mathrm{T}} \mathbf{u}=1 \\
\mathbf{H}^{\mathrm{T}} \mathbf{u}=\sum_{k=1}^{m} \mathbf{C}_{0}^{(k) \mathrm{T}} \boldsymbol{\kappa}_{0}^{(k)}+\sum_{k=1}^{m} \mathbf{C}_{a}^{(k) \mathrm{T}} \mathbf{\kappa}_{a}^{(k)}+\sum_{k=1}^{m} \mathbf{C}_{b}^{(k) \mathrm{T}} \mathbf{\kappa}_{b}^{(k)}
\end{aligned}
$$

where variables are $\mathbf{u}$ and $\left(\kappa_{0}^{(k)}, \boldsymbol{\kappa}_{a}^{(k)}, \boldsymbol{\kappa}_{b}^{(k)}\right)(k=1, \ldots, m)$. Note that the second equality constraint describes strain-displacement relation, and its right-hand side represents generalized member strain vector. Detail of derivation of the dual problem is shown in Appendix.

The matrices $\mathbf{C}_{0}^{(k)}, \mathbf{C}_{a}^{(k)}$, and $\mathbf{C}_{b}^{(k)}$ are condensed to matrices $\mathbf{D}_{0} \in \mathbb{R}^{1 \times 6}, \mathbf{D}_{a} \in \mathbb{R}^{3 \times 6}$, and $\mathbf{D}_{b} \in \mathbb{R}^{3 \times 6}$ corresponding to $\mathbf{f}^{(k)}=\left(N^{(k)}, T^{(k)}, M_{a 2}^{(k)}, M_{a 3}^{(k)}, M_{b 2}^{(k)}, M_{b 3}^{(k)}\right)^{\mathrm{T}}$ with six columns as

$$
\begin{aligned}
\mathbf{D}_{0}=\left(\begin{array}{llllll}
1 & 0 & 0 & 0 & 0 & 0
\end{array}\right), \quad \mathbf{D}_{a}=\left(\begin{array}{cccccc}
0 & -1 & 0 & 0 & 0 & 0 \\
0 & 0 & 1 & 0 & 0 & 0 \\
0 & 0 & 0 & 1 & 0 & 0
\end{array}\right) \\
\mathbf{D}_{b}=\left(\begin{array}{llllll}
0 & 1 & 0 & 0 & 0 & 0 \\
0 & 0 & 0 & 0 & 1 & 0 \\
0 & 0 & 0 & 0 & 0 & 1
\end{array}\right)
\end{aligned}
$$

Hence, from Eq. (2) and the second equality constraint of problem (6), the generalized member strain vector $\mathbf{c}^{(k)}=\left(c_{1}^{(k)}, \ldots, c_{6}^{(k)}\right)^{\mathrm{T}}$ corresponding to $\mathbf{f}^{(k)}$ is given as 
Journal of Mechanical Design

$$
\left(\begin{array}{c}
c_{1}^{(k)} \\
c_{2}^{(k)} \\
c_{3}^{(k)} \\
c_{4}^{(k)} \\
c_{5}^{(k)} \\
c_{6}^{(k)}
\end{array}\right)=\left(\mathbf{D}_{0}^{\mathrm{T}} \kappa_{0}^{(k)}+\mathbf{D}_{a}^{\mathrm{T}} \boldsymbol{\kappa}_{a}^{(k)}+\mathbf{D}_{b}^{\mathrm{T}} \boldsymbol{\kappa}_{b}^{(k)}\right)=\left(\begin{array}{c}
\boldsymbol{\kappa}_{0}^{(k)} \\
-\boldsymbol{\kappa}_{a 1}^{(k)}+\boldsymbol{\kappa}_{b 1}^{(k)} \\
\boldsymbol{\kappa}_{a 2}^{(k)} \\
\boldsymbol{\kappa}_{a 3}^{(k)} \\
\boldsymbol{\kappa}_{b 2}^{(k)} \\
\boldsymbol{\kappa}_{b 3}^{(k)}
\end{array}\right)
$$

where $\kappa_{a i}^{(k)}$ and $\kappa_{b i}^{(k)}$ are the $i$ th components of $\boldsymbol{\kappa}_{a}^{(k)}$ and $\boldsymbol{\kappa}_{b}^{(k)}$, respectively. It is seen from Eq. (8) that the torsional angle $c_{2}^{(k)}$ is defined as the difference between the rotational angles $\kappa_{a 1}^{(k)}$ and $\kappa_{b 1}^{(k)}$ around the member axis at nodes $a$ and $b$. Problem (6) is regarded as a limit analysis problem based on the upper-bound theorem.

By solving the limit analysis problem (5), a collapse mechanism is obtained from the Lagrange multipliers of the constraints, and a linkage mechanism can be generated replacing plastic hinges with revolute joints. Let $n_{\mathrm{h}}$ denote the number of hinges. The conditions for hinge directions are assembled using a $6 m \times n_{\mathrm{h}}$ matrix $\mathbf{R} \in \mathbb{R}^{6 m \times n_{\mathrm{h}}}$ as $\mathbf{R}^{\mathrm{T}} \mathbf{f}=\mathbf{0}$, which leads to the equilibrium equations of the structure with hinges as [17]

$$
\mathbf{G f}=\mathbf{0}, \quad \mathbf{G}=\left(\begin{array}{c}
\mathbf{H} \\
\mathbf{R}^{\mathrm{T}}
\end{array}\right)
$$

Let $g_{\mathrm{r}}$ and $g_{\mathrm{c}}$ denote the numbers of rows and columns, respectively, of matrix $\mathbf{G}$. The rank $r$ of the rectangular matrix $\mathbf{G}$ is computed by carrying out the singular value decomposition. The degrees of statical indeterminacy and kinematical indeterminacy are obtained as $g_{\mathrm{c}}-r$ and $g_{\mathrm{r}}-r$, respectively. Let $\psi$ denote the left singular vector of $\mathbf{G}$ corresponding to zero singular value; i.e., $\left(\mathbf{H}^{\mathrm{T}}, \mathbf{R}\right) \boldsymbol{\psi}=\mathbf{0}$. The vector $\boldsymbol{\psi}$ is divided as 
Journal of Mechanical Design

$\boldsymbol{\psi}=\left(\left(\boldsymbol{\psi}^{\mathrm{u}}\right)^{\mathrm{T}},\left(\boldsymbol{\psi}^{\mathrm{R}}\right)^{\mathrm{T}}\right)^{\mathrm{T}}$, where $\boldsymbol{\psi}^{\mathrm{u}}$ is the vector consisting of the nodal displacements, and $\psi^{\mathrm{R}}$ is the generalized strain vector representing hinge rotations.

Using $\quad \mathbf{f}^{(k)}=\left(N^{(k)}, T^{(k)}, M_{a 2}^{(k)}, M_{a 3}^{(k)}, M_{b 2}^{(k)}, M_{b 3}^{(k)}\right)^{\mathrm{T}}, \quad \mathbf{r}_{a}^{(k)}=\left(r_{a 1}^{(k)}, r_{a 2}^{(k)}, r_{a 3}^{(k)}\right)^{\mathrm{T}} \quad, \quad$ and $\mathbf{r}_{b}^{(k)}=\left(r_{b 1}^{(k)}, r_{b 2}^{(k)}, r_{b 3}^{(k)}\right)^{\mathrm{T}}$, we see that the member-end moments of member $k$ satisfy

$$
\left(\begin{array}{cccccc}
0 & -r_{a 1}^{(k)} & r_{a 2}^{(k)} & r_{a 3}^{(k)} & 0 & 0 \\
0 & r_{b 1}^{(k)} & 0 & 0 & r_{b 2}^{(k)} & r_{b 3}^{(k)}
\end{array}\right) \mathbf{f}^{(k)}=\mathbf{0}
$$

Let $\theta_{a}^{(k)}$ and $\theta_{b}^{(k)}$ denote the components of $\psi$ corresponding to the hinge rotations of nodes $a$ and $b$ of member $k$. Then the rotations of nodes $a$ and $b$ around axes 2 and 3 are obtained as $\theta_{a}^{(k)} r_{a 2}^{(k)}, \theta_{a}^{(k)} r_{a 3}^{(k)}, \theta_{b}^{(k)} r_{b 2}^{(k)}$, and $\theta_{b}^{(k)} r_{b 3}^{(k)}$, and the torsional angle $\Theta^{(k)}$ of member $k$ is computed from

$$
\Theta^{(k)}=-\theta_{a}^{(k)} r_{a 1}^{(k)}+\theta_{b}^{(k)} r_{b 1}^{(k)}
$$

It has been found from our numerical experiments that mechanisms with local deformation near input and/or output nodes are often obtained by simply solving problem (5) or (6). Therefore, additional inequality constraints $\mathbf{A}_{1} \mathbf{u} \geq \mathbf{b}_{1}$ and equality constraints $\mathbf{A}_{2} \mathbf{u}=\mathbf{b}_{2}$ are incorporated into problem (6) as

$$
\begin{aligned}
& \underset{\substack{\left.\mathbf{u}, \kappa_{0}^{(1)}\right), \ldots, \kappa_{0}^{(m)}, \mathbf{\kappa}_{a}^{(1)}, \ldots, \mathbf{K}_{a}^{(m)}, \mathbf{\kappa}_{b}^{(1)}, \ldots, \mathbf{\kappa}_{b}^{(m)}}}{\operatorname{minime}}-\mathbf{p}_{\text {out }}^{\mathrm{T}} \mathbf{u}+\sum_{k=1}^{m} \alpha w_{\mathrm{f}}\left|\kappa_{0}^{(k)}\right|+\sum_{k=1}^{m} \alpha w_{\mathrm{m}}\left\|\mathbf{\kappa}_{a}^{(k)}\right\|+\sum_{k=1}^{m} \alpha w_{\mathrm{m}}\left\|\mathbf{\kappa}_{b}^{(k)}\right\| \\
& \text { subject to } \mathbf{p}_{\text {in }}^{\mathrm{T}} \mathbf{u}=1 \\
& \mathbf{H}^{\mathrm{T}} \mathbf{u}=\sum_{k=1}^{m} \mathbf{C}_{0}^{(k) \mathrm{T}} \boldsymbol{\kappa}_{0}^{(k)}+\sum_{k=1}^{m} \mathbf{C}_{a}^{(k) \mathrm{T}} \boldsymbol{\kappa}_{a}^{(k)}+\sum_{k=1}^{m} \mathbf{C}_{b}^{(k) \mathrm{T}} \boldsymbol{\kappa}_{b}^{(k)} \\
& \mathbf{A}_{1} \mathbf{u} \geq \mathbf{b}_{1} \\
& \mathbf{A}_{2} \mathbf{u}=\mathbf{b}_{2}
\end{aligned}
$$


Journal of Mechanical Design

where variables are $\mathbf{u}$ and $\left(\boldsymbol{\kappa}_{0}^{(k)}, \boldsymbol{\kappa}_{a}^{(k)}, \boldsymbol{\kappa}_{b}^{(k)}\right)(k=1, \ldots, m)$. Concrete examples of these additional constraints appear in the numerical experiments with Models 1 and 3 in Section 4.

The dual problem of problem (12) is obtained as

$$
\begin{array}{cl}
\underset{\lambda_{\text {in }} \mathbf{f}, \mathbf{s}_{1}, \mathbf{s}_{2}}{\operatorname{maximize}} & \lambda_{\text {in }}+\mathbf{b}_{1}^{\mathrm{T}} \mathbf{s}_{1}+\mathbf{b}_{2}^{\mathrm{T}} \mathbf{s}_{2} \\
\text { subject to } & \mathbf{H f}=\mathbf{p}_{\text {out }}+\lambda_{\text {in }} \mathbf{p}_{\text {in }}+\mathbf{A}_{1}^{\mathrm{T}} \mathbf{s}_{1}+\mathbf{A}_{2}^{\mathrm{T}} \mathbf{s}_{2} \\
& \left\|\mathbf{C}_{j}^{(k)} \mathbf{f}\right\| \leq \alpha w_{\mathrm{m}}, \quad(k=1, \ldots, m ; j \in\{a, b\}) \\
& \left|\mathbf{C}_{0}^{(k)} \mathbf{f}\right| \leq \alpha w_{\mathrm{f}}, \quad(k=1, \ldots, m) \\
& \mathbf{s}_{1} \geq \mathbf{0}
\end{array}
$$

where variables are $\lambda_{\text {in }}, \mathbf{f}, \mathbf{s}_{1}$, and $\mathbf{s}_{2}$. Note that the vectors $\mathbf{s}_{1}$ and $\mathbf{s}_{2}$ in problem (13) are regarded as reactions for unilateral and bilateral contact constraints in problem (12).

It should be noted here that only an infinitesimal mechanism is obtained by solving the SOCP problem representing a limit analysis problem. Therefore, large deformation analysis is carried out, and more hinges should be added and more members are removed, if necessary.

\section{NUMERICAL EXAMPLES}

SOCP problems are solved using the optimization library SNOPT Ver. 7.2 [29] to generate mechanisms with inclined joints. In the following examples, a global mechanism of Model 1 that could not be found without heuristics in Ref. 16 is found successfully by solving problem (12) with displacement constraints. It is confirmed using Model 2 that the same solution is obtained by solving problems (5) and (6). Additional constraints are 
Journal of Mechanical Design

given as problem (12) for Model 3. The units of force and length are not written explicitly, because the models can be scaled arbitrarily. The weight coefficients $w_{\mathrm{m}}$ and $w_{\mathrm{f}}$ are 1.0 and 10.0, respectively, for both of the following examples.

Abaqus Ver. 6.14 [30] is used for investigating the properties in large-deformation range by increasing the path parameter $t$ from 0 to 1 corresponding to initial and final configurations, respectively. The zero-length element called CONN3D2 is adopted for a revolute joint. The units of force and length are shown in the results of geometrically nonlinear analysis.

\section{Model 1}

Consider a plane grid model as shown in Fig. 2 to demonstrate the effectiveness of the proposed method. This model was investigated in Ref. [16] using a linear programming approach. A mechanism is to be found so that node B moves upward as a result of pulling node A downward 1.0. However, a local mode as shown in Fig. 3(a) with displacements only at node B is found by solving an LP problem. A filled circle in the figure indicates a hinge at a member end. A global mechanism could be obtained with explicit assignment of existing members ' $a$ ', ' $b$ ', ' $c$ ', and 'd' in Fig. 2.

The same problem is solved using an SOCP problem (12) with parameter value $\alpha=0.035$, which has been obtained using the same approach as Ref. [16]. If problem (12) is solved, then a local mode without displacement of node B is obtained as shown in Fig. 3(b). Therefore, displacement constraints are assigned so that the horizontal displacements of nodes $C$ and $D$ toward the center node $O$ is greater than 1.0. The 
Journal of Mechanical Design

mechanism obtained with the displacement constraints is shown in Fig. 3(c). This way, a global mode is found by solving an SOCP problem. However, the mechanism in Fig. 3(c) is only assured to be an infinitesimal mechanism. Therefore, a large deformation analysis is carried out to investigate its property in finite deformation range. We found that an external force is needed for large deformation of the mechanism in Fig. 3(c). It is natural to remove members with largest axial force to obtain a finite mechanism. This way, a finite mechanism as shown in Fig. 3(d) has been obtained.

\section{Model 2}

Consider a square grid with unit member length as shown in Fig. 4(a). Nodes 2 and 4 are supported in $y$-and $z$-directions, and nodes 3 and 5 are supported in $x$ - and $z$-directions [16-18].

The input and output loads are given as shown in Fig. 4(b) to generate a mechanism so that the output nodes $6-9$ move in $z$-direction as a result of pulling the input node 1 in negative z-direction. The primal problem (5) and the dual problem (6) are solved for $\alpha=0.37$, which is decided using the procedure in Ref. [16]. We confirmed that the same hinge locations are found by solving the primal and dual problems. However, the number of hinges is too small due to strong symmetry of the model. Therefore, the $z$ coordinate of node 1 is decreased by 0.01 , and problems (5) and (6) are solved again.

Figure 5 illustrates the locations and directions of hinges. As seen in the figure, the hinges of members $1-4$ are directed to local axis 2 . Table 1 shows the hinge directions of member 5 at the connections to nodes 2 and 6 obtained by solving the primal and dual 
Journal of Mechanical Design

problems. It is confirmed that the ratios among $x-, y$-, and $z$-components of direction vectors are almost the same for primal and dual problems.

Large-deformation analysis is carried out to find that the reaction force at the input node 1 is not zero. In a manner similar to Ref. [16-18], we added eight torsional hinges as indicated with $\times$ in Fig. 6 to generate a finite mechanism that has no reaction force in the deformation process. The deformation process of the finite mechanism is shown in Fig. 7.

\section{Model 3}

We next consider a plane frame consisting of two square grids as shown in Fig. 8(a). Nodes 2 and 4 are supported in $y$-and $z$-directions, and nodes 3 and 5 are supported in $x$ - and $z$-directions. A mechanism is generated so that the output nodes $6,7,8$, and 9 move in $z$-direction and nodes $14,15,16$, and 17 move in negative $z$-direction as a result of pulling the input node 1 in $z$-direction. Therefore, the input and output loads are applied as shown in Fig. 8(b).

By solving problem (6), a mechanism is found in which nodes $6,7,8$, and 9 do not move in $z$-direction. Therefore, problem (12) with additional constraints is solved; accordingly, the procedure in Ref. [16] for deciding an appropriate value of $\alpha$ cannot be used. As observed from the definition of objective and constraint functions of problem (12), the displacements become very small and output displacements vanish if $\alpha$ is too large. By contrast, the problem becomes unbounded below, if $\alpha$ is too small. We found appropriate value $\alpha=1.2$ after several trials. 
Journal of Mechanical Design

We solve problem (12) assigning a lower-bound displacement 0.5 for nodes $6,7,8$, and 9 , and an upper-bound displacement -0.5 for nodes $14,15,16$, and 17 . Equality constraints are also given so that the $z$-directional displacements of three sets of nodes $\{6,7,8,9\},\{10,11,12,13\}$, and $\{14,15,16,17\}$ have the same value, respectively. The hinge locations of the solution of problem (12) are shown in Fig. 9. At the optimal solution, the inequality constraints on the displacements at nodes $6,7,8$, and 9 become active, while the ones on the displacements at nodes $14,15,16$, and 17 are inactive.

The degrees of kinematical and statical indeterminacies obtained by the singular value decomposition of $\mathbf{G}$ are 1 and 7, respectively. Large-deformation analysis is carried out by assigning forced displacement in negative $z$-direction at node 1 . The deformation process is shown in Fig. 10. We confirmed that no force is needed for generating this large deformation. Fig. 11 shows the relation between the path parameter and the displacements at nodes 6 and 14 .

It should be noted here that the lower-bound displacements are given to obtain a mechanism that deforms in the desired direction, and the deformation shape is constrained by the lengths of members. We confirmed that the same large deformation property is obtained from the infinitesimal mechanisms generated with the lowerbound displacements $0.3,0.4,0.5,0.6$, and 0.7 .

\section{CONCLUSIONS}

A new method has been presented for generating a mechanism with inclined hinges by solving a primal-dual pair of SOCP problems. When the obtained mechanism has local 
Journal of Mechanical Design

deformation near the input and/or output nodes, inequality constraints can be added, to the problem based on the upper-bound theorem, to generate a mechanism with global deformation. It is seen from its dual problem that the Lagrange multiplier for a displacement constraint corresponds to an additional reaction nodal force. It should be noted that it is necessary to formulate the problem to an SOCP problem to clearly separate the force variables and displacement and strain variables.

The kinematic indeterminacy of the mechanism can be evaluated by the singular value decomposition of a matrix defining equilibrium conditions and hinge directions. It is confirmed that the torsional angle of a member is obtained as the difference between the rotations around the member axis at the two ends of the member.

It has been demonstrated in the numerical examples that the global mechanism of plane grid model, which could not be found without an ad hoc process if the method in Ref. [16] was used, can be found using the proposed method with explicit assignment of displacement constraints.

\section{REFERENCES}

1. L. W. Tsai: Mechanism Design: Enumeration of Kinematic Structures According to Function. CRC Press, Boca Raton, 2001.

2. C. J. Gantes: Deployable Structures: Analysis and Design. WIT Press, 2001.

3. S. Pellegrino (Ed.): Deployable Structures. CISM Courses and Lectures, No. 412, Springer-Verlag, Wien, 2001.

4. L. A. Mira, R. F. Coelho, A. P. Thrall and N. De Temmerman: Parametric evaluation of 
Journal of Mechanical Design

deployable scissor arches. Engineering Structures, Vol. 99, pp. 479-491, 2015.

5. Y. Akgün, C. J. Gantes, W. Sobek, K. Korkmaz and K. Kalochairetis: A novel adaptive spatial scissor-hinge structural mechanism for convertible roofs. Engineering Structures, Vol. 33, pp. 1365-1376, 2011.

6. R. R. Root and K. M. Ragsdell: A survey of optimization methods applied to the design of mechanisms. Journal of Engineering for Industry (ASME), Vol. 98, pp. 1036-1041, 1976.

7. M. Stolpe and A. Kawamoto: Design of planar articulated mechanisms using branch and bound. Mathematical Programming, Vol. 103, pp. 357-397, 2005.

8. Y. Liu and J. McPhee. Automated type synthesis of planar mechanisms using numeric optimization with genetic algorithms. Journal of Mechanical Design (ASME), Vol. 127, pp. 910-916, 2004.

9. Y. Y. Kim, G.-W. Jang, J. H. Park, J. S. Hyun and S. J. Nam: Automatic synthesis of a planar linkage mechanism with revolute joints by using spring-connected rigid block models. Journal of Mechanical Design (ASME), Vol. 129, pp. 930-940, 2007.

10. M. Ohsaki and S. Nishiwaki: Generation of link mechanism by shape-topology optimization of trusses considering geometrical nonlinearity. Journal of Computational Science and Technology (JSME), Vol. 3, pp. 46-53, 2009.

11. S. W. Kang, S. I. Kim and Y. Y. Kim: Topology optimization of planar linkage systems involving general joint types. Mechanism and Machine Theory, Vol. 104, pp. 130160, 2016.

12. N. Katoh and S. Tanigawa: On the infinitesimal rigidity of bar-and-slider frameworks. 
Journal of Mechanical Design

In: Y. Dong, D.-Z, Du and O. Ibarra (eds.), Algorithms and Computation, Lecture Notes in Computer Science, Volume 5878, Springer-Verlag, Berlin, pp. 524-533, 2009.

13. S. M. Han, S. I. Kim and Y. Y. Kim, Topology optimization of planar linkage mechanisms for path generation without prescribed timing, Structural and Multidisciplinary Optimization, Vol. 56, pp. 501-517, 2017.

14. S. I. Kim, S. W. Kang, Y.-S. Yi, J. Park and Y. Y. Kim, Topology optimization of vehicle rear suspension mechanisms, International Journal for Numerical Methods in Engineering, Vol. 113, pp. 1412-1433, 2017.

15. S. I. Kim and Y. Y. Kim, Topology optimization of planar linkage mechanism, International Journal for Numerical Methods in Engineering, Vol. 98, pp. 265-286, 2014.

16. M. Ohsaki, Y. Kanno Y. and S. Tsuda: Linear programming approach to design of spatial link mechanism with partially rigid joints. Structural and Multidisciplinary Optimization, Vol. 50, pp. 945-956, 2014.

17. S. Tsuda, M. Ohsaki, S. Kikugawa and Y. Kanno: Analysis of stability and mechanism of frames with partially rigid connections. Journal of Structural and Construction Engineering (Transactions of AIJ), Vol. 78, No. 686, pp. 791-798, 2013. (in Japanese)

18. M. Ohsaki, S. Tsuda and Y. Miyazu: Design of linkage mechanisms of partially rigid frames using limit analysis with quadratic yield functions. International Journal of Solids and Structures, Vol. 88-89, pp. 68-78, 2016.

19. M. F. Anjos and J. B. Lasserre (eds.): Handbook on Semidefinite, Conic and 
Journal of Mechanical Design

Polynomial Optimization. Springer, New York (2012).

20. F. Alizadeh and D. Goldfarb: Second-order cone programming. Mathematical Programming, Vol. 95, pp. 3-51, 2003.

21. C. D. Bisbos, A. Makrodimopoulos and P. M. Pardalos: Second-order cone programming approaches to static shakedown analysis in steel plasticity. Optimization Methods and Software, Vol. 20, pp. 25-52, 2005.

22. A. Makrodimopoulos and C. M. Martin: Lower bound limit analysis of cohesivefrictional materials using second-order cone programming. International Journal for Numerical Methods in Engineering, Vol. 66, pp. 604-634, 2006.

23. K. Krabbenhøft, A. V. Lyamin and S. W. Sloan: Formulation and solution of some plasticity problems as conic programs. International Journal of Solids and Structures, Vol. 44, pp. 1533-1549, 2007.

24. J. Pastor, Ph. Thoré and F. Pastor: Limit analysis and numerical modeling of spherically porous solids with Coulomb and Drucker-Prager matrices. Journal of Computational and Applied Mathematics, Vol. 234, pp. 2162-2174, 2010.

25. J. Bleyer, M. Maillard, P. de Buhan and P. Coussot: Efficient numerical computations of yield stress fluid flows using second-order cone programming. Computer Methods in Applied Mechanics and Engineering, Vol. 283, pp. 599-614, 2015.

26. K. Yonekura and Y. Kanno: Second-order cone programming with warm start for elastoplastic analysis with von Mises yield criterion. Optimization and Engineering, Vol. 13, pp. 181-218, 2012.

27. Y. Kanno and M. Ohsaki: Minimum principle of complementary energy for nonlinear 
Journal of Mechanical Design

elastic cable networks with geometrical nonlinearity. Journal of Optimization Theory and Applications, Vol. 126(3), pp. 617-641, 2005.

28. Y. Kanno, M. Ohsaki and J. Ito: Large-deformation and friction analysis of nonlinear elastic cable networks by second-order cone programming. International Journal for Numerical Methods in Engineering, Vol. 55, pp. 1079-1114, 2002.

29. P. E. Gill, W. Murray and M. A. Saunders: SNOPT: An SQP algorithm for large-scale constrained optimization. SIAM Journal on Optimization, Vol. 12, pp. 979-1006, 2002.

30. Dassault Systèmes: Abaqus User's Manual Ver. 6.14, 2014. 
Journal of Mechanical Design

\section{APPENDIX: LAGRANGE DUAL PROBLEM OF (5)}

Problem (6) can be derived as the Lagrange dual problem of (5). We briefly outline the derivation in this appendix.

We first observe that the Lagrangian of problem (5) can be formulated as

$$
\begin{aligned}
& L\left(\lambda_{\text {in }}, \mathbf{f} ; \mathbf{u}, \boldsymbol{\mu}, \mathbf{\kappa}\right) \\
& =\left\{\begin{array}{l}
\lambda_{\text {in }}+\mathbf{u}^{\mathrm{T}}\left(\mathbf{H} \mathbf{f}-\mathbf{p}_{\text {out }}-\lambda_{\text {in }} \mathbf{p}_{\text {in }}\right) \\
+\sum_{j \in\{a, b\}} \sum_{k=1}^{m}\left[\begin{array}{l}
\mu_{j}^{(k)} \\
\mathbf{\kappa}_{j}^{(k)}
\end{array}\right]\left[\begin{array}{l}
\alpha w_{\mathrm{m}} \\
\mathbf{C}_{j}^{(k)} \mathbf{f}
\end{array}\right] \\
+\sum_{k=1}^{m}\left[\begin{array}{c}
\mu_{0}^{(k)} \\
\boldsymbol{\kappa}_{0}^{(k)}
\end{array}\right]^{\top}\left[\begin{array}{c}
\alpha w_{\mathrm{f}} \\
\mathbf{C}_{0}^{(k)} \mathbf{f}
\end{array}\right] \text { if } \mu_{j}^{(k)} \geq\left\|\boldsymbol{\kappa}_{j}^{(k)}\right\|(k=1, \ldots, m ; \forall j \in\{a, b\}) \\
-\infty \quad \text { otherwise }
\end{array}\right.
\end{aligned}
$$

where $\mathbf{u} \in \mathbb{R}^{n}, \mu_{a}^{(k)} \in \mathbb{R}, \mu_{b}^{(k)} \in \mathbb{R}, \mu_{0}^{(k)} \in \mathbb{R}, \boldsymbol{\kappa}_{a}^{(k)} \in \mathbb{R}^{3}, \mathbf{\kappa}_{b}^{(k)} \in \mathbb{R}^{3}$, and $\kappa_{0}^{(k)} \in \mathbb{R}$ $(k=1, \ldots, m)$ are the Lagrange multipliers, and for simplicity of notation we write

$$
\begin{aligned}
& \boldsymbol{\mu}=\left(\mu_{a}^{(1)}, \ldots, \mu_{a}^{(m)}, \mu_{b}^{(1)}, \ldots, \mu_{b}^{(m)}, \mu_{0}^{(1)}, \ldots, \mu_{0}^{(m)}\right), \\
& \mathbf{\kappa}=\left(\boldsymbol{\kappa}_{a}^{(1)}, \ldots, \boldsymbol{\kappa}_{a}^{(m)}, \boldsymbol{\kappa}_{b}^{(1)}, \ldots, \boldsymbol{\kappa}_{b}^{(m)}, \boldsymbol{\kappa}_{0}^{(1)}, \ldots, \boldsymbol{\kappa}_{0}^{(m)}\right)
\end{aligned}
$$

Indeed, a short calculation yields

$$
\begin{aligned}
& \inf \left\{L\left(\lambda_{\text {in }}, \mathbf{f} ; \mathbf{u}, \boldsymbol{\mu}, \boldsymbol{\kappa}\right) \mid(\mathbf{u}, \boldsymbol{\mu}, \boldsymbol{\kappa}) \in \Omega_{\mathrm{D}}\right\} \\
& =\left\{\begin{array}{c}
\lambda_{\text {in }} \text { if } \quad \mathbf{H f}=\mathbf{p}_{\text {in }}+\lambda_{\text {in }} \mathbf{p}_{\text {out }}, \\
\alpha w_{\mathrm{m}} \geq\left\|\mathbf{C}_{j}^{(k)} \mathbf{f}\right\|, \quad(k=1, \ldots, m ; \forall j \in\{a, b\}), \\
\alpha w_{\mathrm{f}} \geq\left|\mathbf{C}_{0}^{(k)} \mathbf{f}\right|, \quad(k=1, \ldots, m) \\
-\infty \quad \text { otherwise }
\end{array}\right.
\end{aligned}
$$

from which we can confirm that problem (5) is equivalently rewritten as

$$
\operatorname{maximize} \inf \left\{L\left(\lambda_{\text {in }}, \mathbf{f} ; \mathbf{u}, \boldsymbol{\mu}, \boldsymbol{\kappa}\right) \mid(\mathbf{u}, \boldsymbol{\mu}, \boldsymbol{\kappa}) \in \Omega_{\mathrm{D}}\right\}
$$


Journal of Mechanical Design

Here, for simplicity of notation, we have used $\Omega_{\mathrm{D}}$ defined by

$$
\begin{gathered}
\Omega_{\mathrm{D}}=\left\{(\mathbf{u}, \boldsymbol{\mu}, \mathbf{\kappa}) \in \mathbb{R}^{n} \times \mathbb{R}^{3 m} \times \mathbb{R}^{7 m} \mid \mu_{j}^{(k)} \geq\left\|\boldsymbol{\kappa}_{j}^{(k)}\right\|, \quad(k=1, \ldots, m ; \forall j \in\{a, b\}),\right. \\
\left.\mu_{0}^{(k)} \geq\left|\kappa_{0}^{(k)}\right|, \quad(k=1, \ldots, m)\right\}
\end{gathered}
$$

The Lagrange dual problem is defined as

$$
\operatorname{minimize} \sup \left\{L\left(\lambda_{\text {in }}, \mathbf{f} ; \mathbf{u}, \boldsymbol{\mu}, \mathbf{\kappa}\right) \mid\left(\lambda_{\text {in }}, \mathbf{f}\right) \in \Omega_{\mathrm{P}}\right\}
$$

where $\Omega_{\mathrm{P}}$ denotes the feasible set of problem (5), i.e.,

$$
\begin{aligned}
\Omega_{\mathrm{P}}=\left\{\left(\lambda_{\text {in }}, \mathbf{f}\right) \in \mathbb{R} \times \mathbb{R}^{n} \mid\right. & \mathbf{H f}=\mathbf{p}_{\text {in }}+\lambda_{\text {in }} \mathbf{p}_{\text {out }}, \\
& \alpha w_{\mathrm{m}} \geq\left\|\mathbf{C}_{j}^{(k)} \mathbf{f}\right\| \quad(k=1, \ldots, m ; \forall j \in\{a, b\}), \\
& \left.\alpha w_{\mathrm{f}} \geq\left|\mathbf{C}_{0}^{(k)} \mathbf{f}\right| \quad(k=1, \ldots, m)\right\}
\end{aligned}
$$

A short calculation shows

$$
\begin{aligned}
& \sup \left\{L\left(\lambda_{\text {in }}, \mathbf{f} ; \mathbf{u}, \boldsymbol{\mu}, \mathbf{\kappa}\right) \mid\left(\lambda_{\text {in }}, \mathbf{f}\right) \in \Omega_{\mathrm{P}}\right\} \\
& = \begin{cases}-\mathbf{p}_{\text {out }}^{\mathrm{T}} \mathbf{u}+\sum_{j \in\{a, b\}} \sum_{k=1}^{m} \alpha w_{\mathrm{m}} \mu_{j}^{(k)} & \\
+\sum_{k=1}^{m} \alpha w_{\mathrm{f}} \mu_{0}^{(k)} & \text { if } \quad \mathbf{p}_{\text {in }}^{\mathrm{T}} \mathbf{u}=1, \\
\mathbf{H}^{\mathrm{T}} \mathbf{u}=\sum_{j \in\{a, b\}} \sum_{k=1}^{m} \mathbf{C}_{j}^{(k) \mathrm{T}} \boldsymbol{\kappa}_{j}^{(k)}+\sum_{k=1}^{m} \mathbf{C}_{0}^{(k) \mathrm{T}} \boldsymbol{\kappa}_{0}^{(k)}, \\
\mu_{j}^{(k)} \geq\left\|\mathbf{\kappa}_{j}^{(k)}\right\|, \quad(k=1, \ldots, m ; \forall j \in\{a, b\}), \\
\mu_{0}^{(k)} \geq\left|\boldsymbol{\kappa}_{0}^{(k)}\right|, \quad(k=1, \ldots, m) \\
\text { otherwise }\end{cases}
\end{aligned}
$$

from which it follows that (A6) is reduced to an explicit form 
Journal of Mechanical Design

$$
\begin{array}{ll}
\operatorname{minimize} & -\mathbf{p}_{\text {out }}^{\mathrm{T}} \mathbf{u}+\sum_{j \in\{a, b\}} \sum_{k=1}^{m} \alpha w_{\mathrm{m}} \mu_{j}^{(k)}+\sum_{k=1}^{m} \alpha w_{\mathrm{f}} \mu_{0}^{(k)} \\
\text { subject to } \quad \mathbf{p}_{\text {in }}^{\mathrm{T}} \mathbf{u} & =1, \\
\mathbf{H}^{\mathrm{T}} \mathbf{u} & =\sum_{j \in\{a, b\}} \sum_{k=1}^{m} \mathbf{C}_{j}^{(k) \mathrm{T}} \mathbf{\kappa}_{j}^{(k)}+\sum_{k=1}^{m} \mathbf{C}_{0}^{(k) \mathrm{T}} \boldsymbol{\kappa}_{0}^{(k)}, \\
\mu_{j}^{(k)} \geq\left\|\mathbf{\kappa}_{j}^{(k)}\right\|, \quad(k=1, \ldots, m ; \forall j \in\{a, b\}), \\
\mu_{0}^{(k)} \geq\left|\boldsymbol{\kappa}_{0}^{(k)}\right|, \quad(k=1, \ldots, m)
\end{array}
$$

Since all the inequality constraints of problem (A9) become active at any optimal solution, we can eliminate variables $\mu_{a}^{(k)}, \mu_{b}^{(k)}$, and $\mu_{0}^{(k)}(k=1, \ldots, m)$ to obtain problem (6). 


\section{Figure Captions List}

Fig. 1 Definition of member coordinates and independent member-end forces; (a) local and global coordinates, (b) six independent member-end forces.

Fig. 2

Fig. 3

Fig. 4

Fig. 5

Fig. 6 torsional hinges.

Fig. 7 Deformation process of finite mechanism of Model 2.

Fig. 8 A plane grid consisting of two square grids (Model 3); (a) node and element indices, (b) input and output loads. Fig. 9 Locations of hinges of Model 3; infinitesimal mechanism obtained by

Fig. 3. Mechanisms of the plane grid model obtained by solving various types of optimization problem; filled circle indicates a hinge; (a) linear programming problem [16], (b) problem (12) without displacement constraints, (c) problem (12) with displacement constraints, (d) finite mechanism obtained by removing four members of infinitesimal mechanism.

A simple square-grid model (Model 2); (a) plan view and node/member indices, (b) diagonal view and input/output loads.

Locations and directions of hinges of Model 2; an infinitesimal mechanism is obtained by solving problems (5) and (6).

Locations of hinges of Model 2; finite mechanism after adding eight 
Journal of Mechanical Design

solving problem (12).

Fig. 10 Deformation process of finite mechanism of Model 3; (a) diagonal view, (b) elevation.

Fig. 11 Relation between the path parameter and the displacements at nodes 6 and 14 of Model 3; solid line: node 6, dashed line: node 14. 
Journal of Mechanical Design

\section{Table Caption List}

Table 1 Directions of hinges in global coordinate system at the connection of member 5 to nodes 2 and 6 of Model 3. 
Journal of Mechanical Design

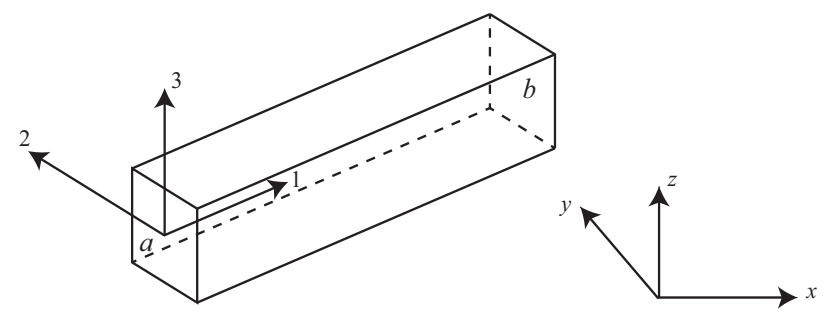

(a)

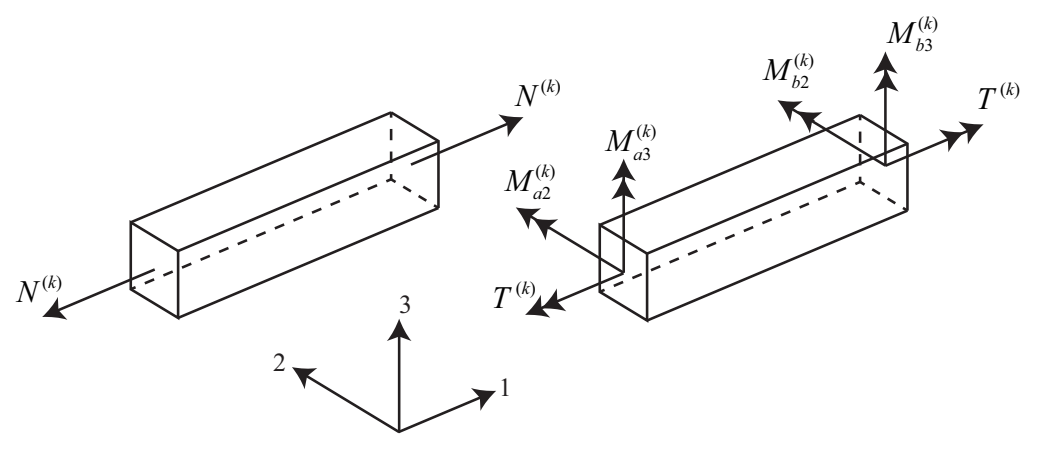

(b)

Figure 1: Definition of member coordinates and independent member-end forces;

(a) local and global coordinates, (b) six independent member-end forces. 
Journal of Mechanical Design

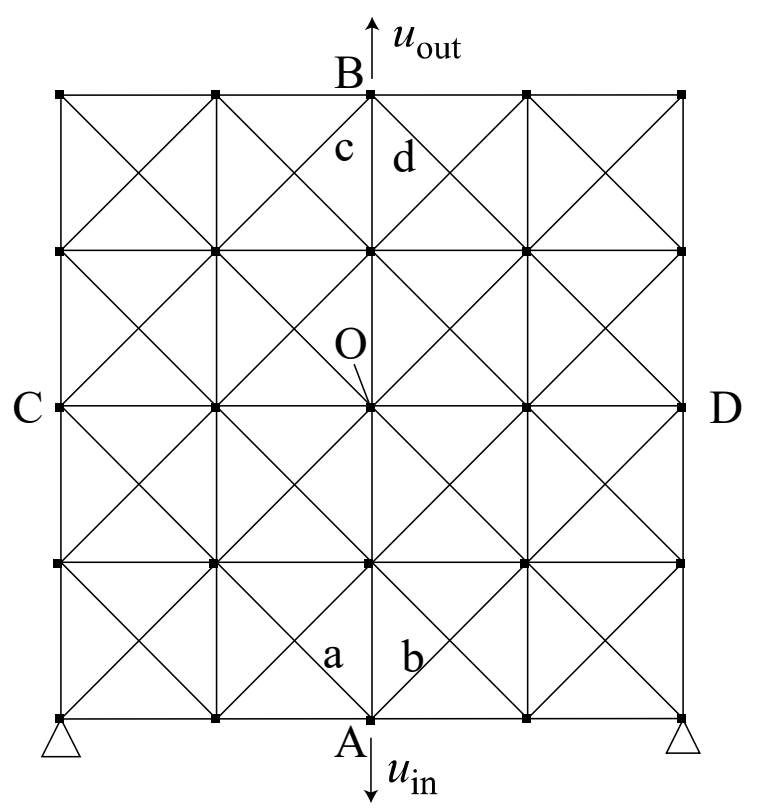

Fig. 2. A plane grid model (Model 1). 


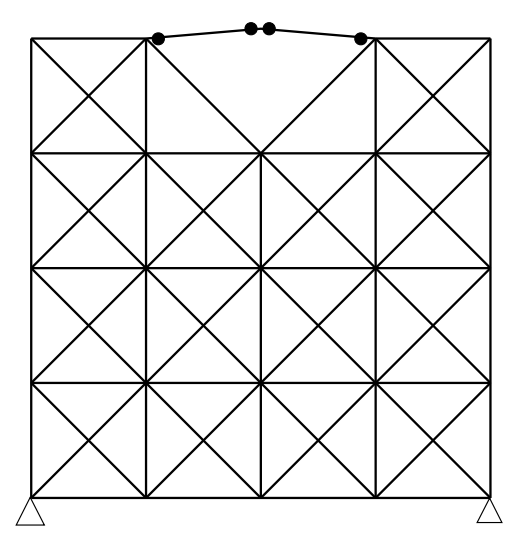

(a)

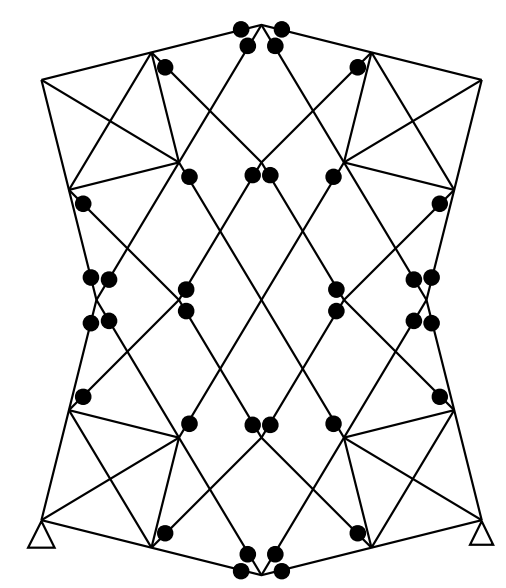

(c)

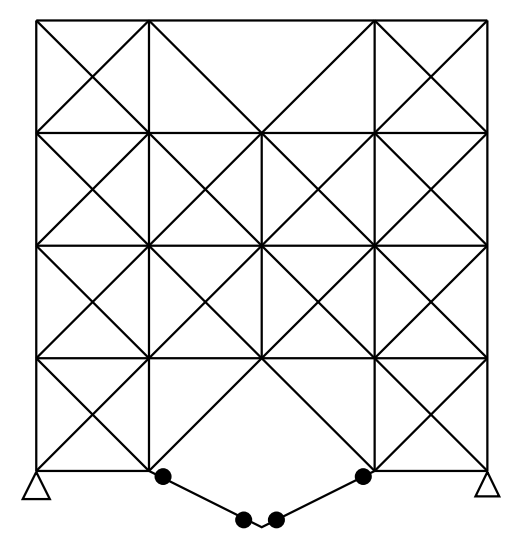

(b)

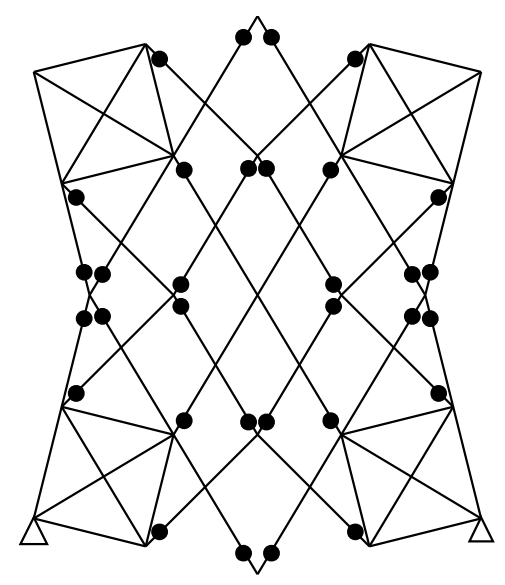

(d)

Fig. 3. Mechanisms of the plane grid model obtained by solving various types of optimization problem; filled circle indicates a hinge; (a) linear programming problem [16], (b) problem (12) without displacement constraints, (c) problem (12) with displacement constraints, (d) finite mechanism obtained by removing four members of infinitesimal mechanism. 
Journal of Mechanical Design

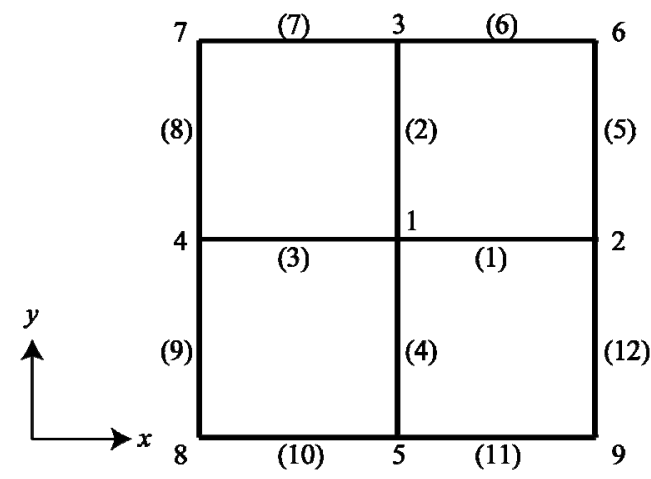

(a)

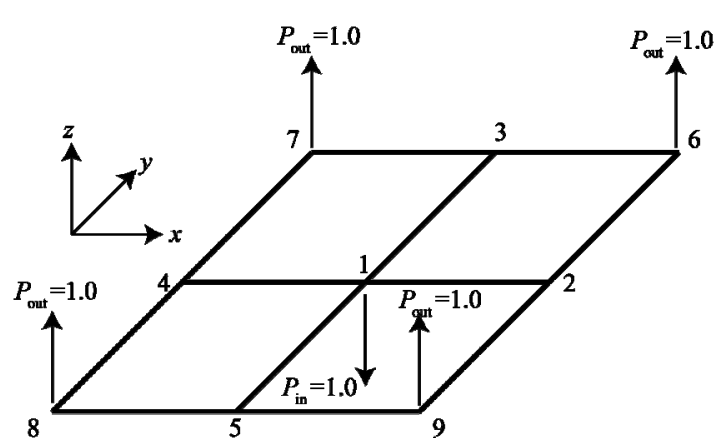

(b)

Figure 4: A simple square-grid model (Model 2); (a) plan view and node/member indices,

(b) diagonal view and input/output loads. 
Journal of Mechanical Design

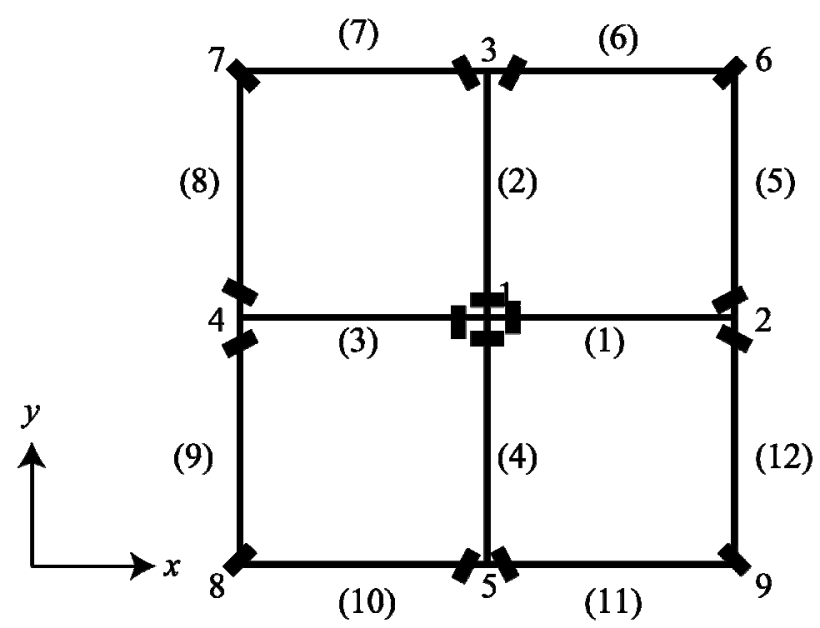

Figure 5: Locations and directions of hinges of Model 2; an infinitesimal mechanism is obtained by solving problems (5) and (6). 
Journal of Mechanical Design

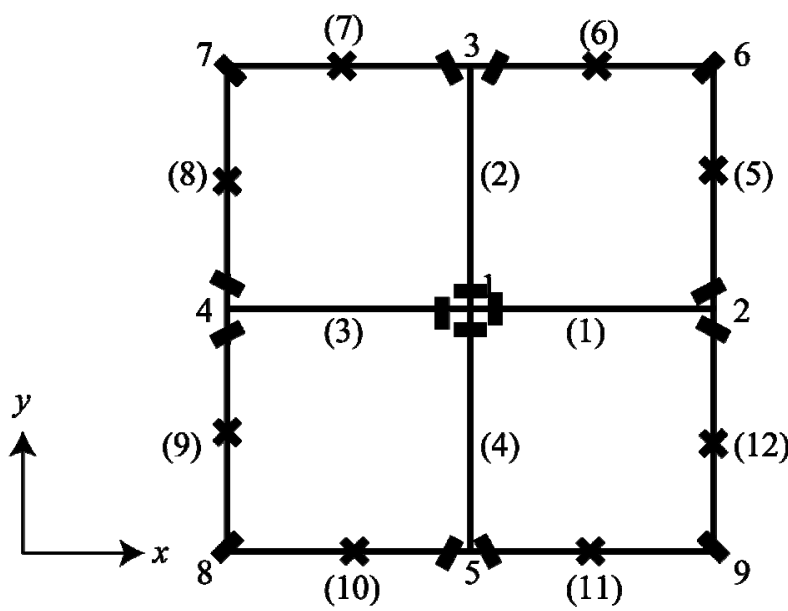

Figure 6: Locations of hinges of Model 2; finite mechanism after adding eight torsional hinges. 

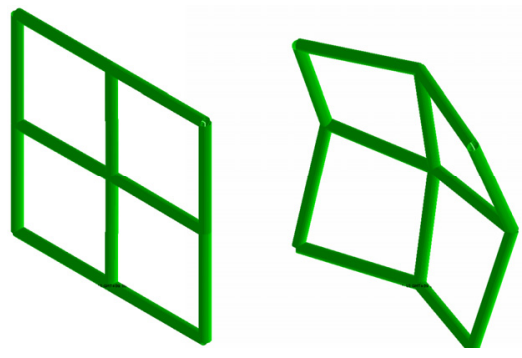

$t=0.0$

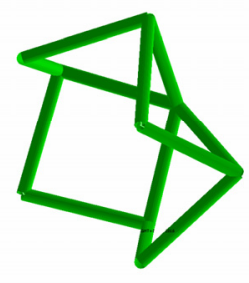

$t=0.30$

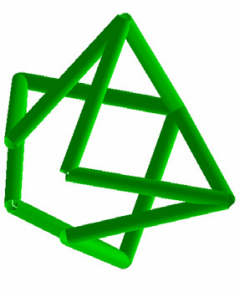

$t=0.45$

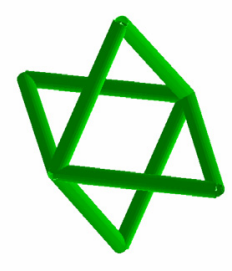

$t=0.56$

Figure 7: Deformation process of finite mechanism of Model 2. 
Journal of Mechanical Design

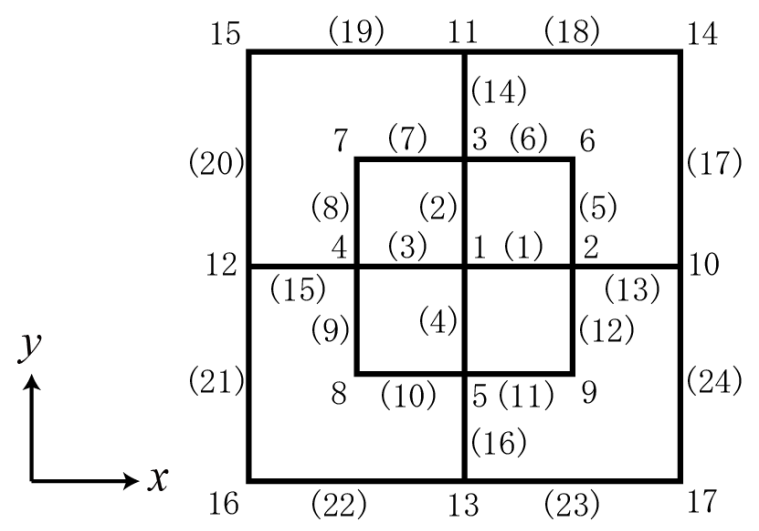

(a)

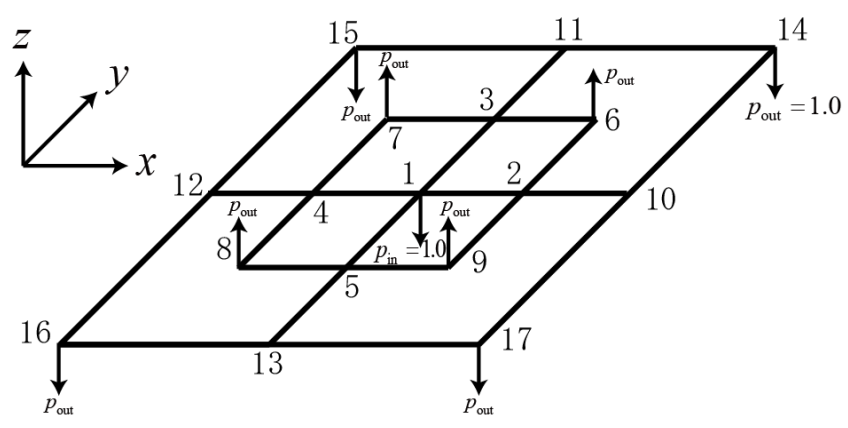

(b)

Figure 8: A plane grid consisting of two square grids (Model 3); (a) node and element indices, (b) input and output loads. 
Journal of Mechanical Design

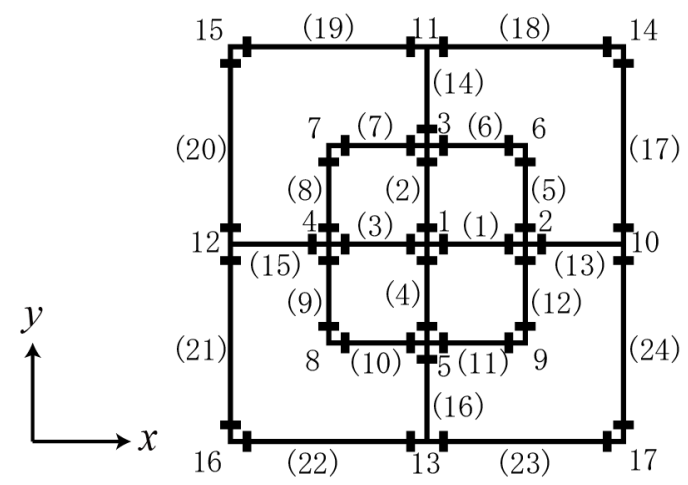

Figure 9: Locations of hinges of Model 3; infinitesimal mechanism obtained by solving problem (12). 


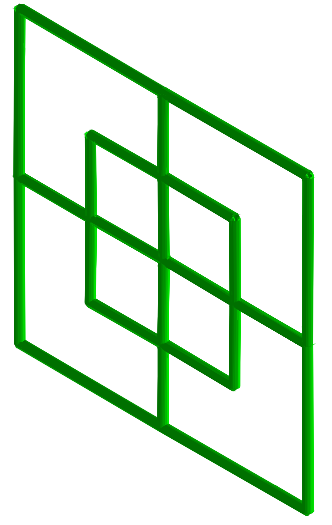

$t=0.0$

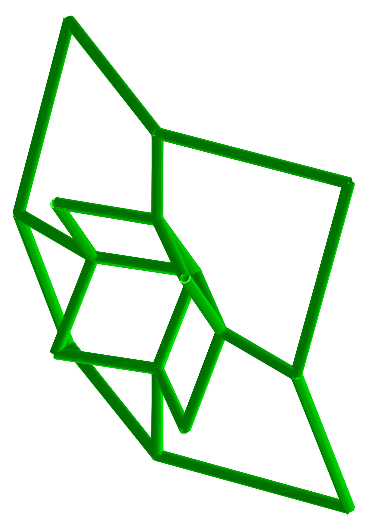

$t=0.5$

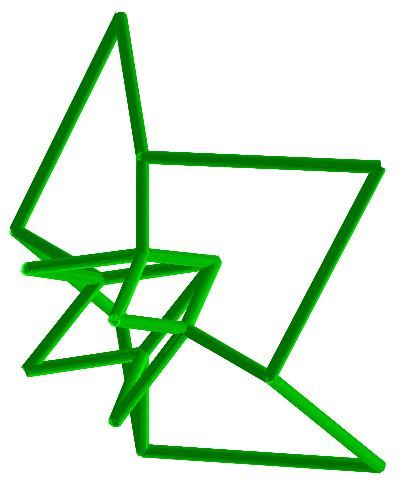

$t=0.85$

(a)

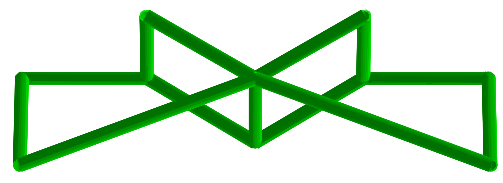

$t=0.5$

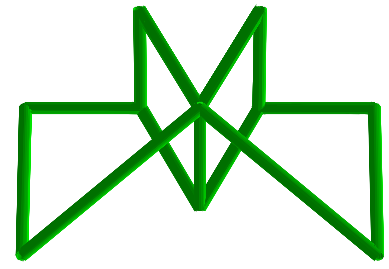

$t=0.85$

(b)

Figure 10: Deformation process of finite mechanism of Model 3; (a) diagonal view,

(b) elevation. 
Journal of Mechanical Design

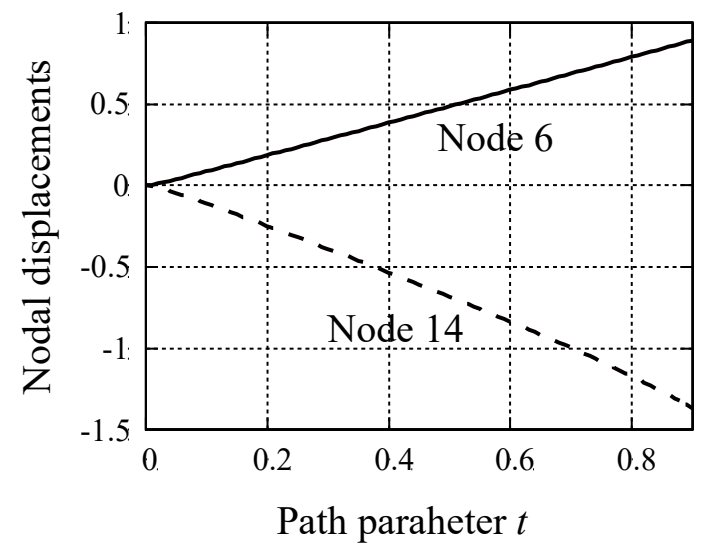

Figure 11: Relation between the path parameter $t$ and the $z$-directional displacements at nodes 6 and 14 of Model 3; solid line: node 6, dashed line: node 14. 
Journal of Mechanical Design

Table 1: Directions of hinges in global coordinate system at the connection of member 5 to nodes 2 and 6 of Model 3.

\begin{tabular}{c|ccccc|ccccc}
\hline & \multicolumn{9}{|c|}{ Node 2 (node $a$ of member 5) } & \multicolumn{5}{|c}{ Node 6 (node $b$ of member 5) } \\
& $x$ & $y$ & $z$ & $y / x$ & $z / x$ & $x$ & $y$ & $z$ & $y / x$ & $z / x$ \\
\hline Problem (5) & 0.173 & 0.173 & 0.277 & 1.00 & -1.61 & -0.327 & 0.173 & 0.0015 & -0.53 & 0.00 \\
Problem (6) & 0.0076 & 0.0075 & 0.0122 & 0.99 & -1.61 & -2.156 & 1.123 & 0.0092 & -0.52 & 0.00 \\
& & & & & & & & & & \\
\hline
\end{tabular}

